\title{
Metabolitos aislados de raputia heptaphylla y esenbeckia alata (rutaceae) y síntesis de precursores de análogos de alcaloides quinolínicos
}

\section{Isolated Metabolites of Raputia Heptaphylla and Esenbeckia Alata (Rutaceae) and Synthesis of Quinolinic Alkaloid Analog Precursors}

\author{
Carlos A. Coy Barrera ${ }^{1 \star}$, Luis E. Cuca Suárez ${ }^{2}$ \\ 1 Universidad Militar Nueva Granada, Facultad de Ciencias Básicas y Aplicadas, \\ Departamento de Química. Bogotá Colombia. \\ ${ }^{2}$ Laboratorio de Investigación en Productos Naturales Vegetales, Universidad Nacional \\ de Colombia, Facultad de Ciencias, Departamento de Química, Bogotá, Colombia.
}

FeCha DE ENTREGa: 9 DE JUNio DE 2014

FECHA DE EVALUACIÓN: 17 DE SEPTIEMBRE DE 2014

FECha dE APROBACIÓN: 23 DE SEPTIEMBRE DE 2014

\begin{abstract}
Resumen Estudios anteriores sobre la esenbeckia alata y la raputia heptaphylla, ambas especies pertenecientes a la familia de las rutáceas, han mostrado resultados importantes sobre su composición química. Estas especies tienen metabolitos secundarios (principalmente cumarinas y alcaloides) que actúan como marcadores quimiotaxonómicos. Este artículo presenta, por primera vez, el trabajo fitoquímico y los metabolitos aislados de estas especies: cuatro cumarinas: bergapteno, xantiletina, xantotoxina, 3-isoprenilo-4-metoxilo-cumarina; cuatro alcaloides: skimmianina, kokusaginina, dictamnina y 1-metil-2- metoxi-4-quinolona; dos lignanos: sesamin y ácido mesodihidroguaiarético; dos esteroles: $\beta$-sitosterol, estigmasterol y un triterpeno pentacíclico: lupeol, los cuales se aislaron de las hojas de E. alata. Cinco alcaloides 7-metoxi-2,2-dimetil-2,6-dihidro-piran[3,2,c] quinolona-5-uno, flindersiamina, skimmianina, kokusaginina y dictamnina, que se aislaron de las hojas de $R$. heptaphylla. Además, el artículo presenta también un nuevo método mediante la obtención de precursores análogos de alcaloides quinolínicos por medio de condensaciones de Mannich aducto vinílogo y aldehídos seguidos por una ciclación radical para obtener productos con alta regio- y estereoselectividad.
\end{abstract}

Abstract Previous studies on the Esenbeckia alata and Raputia heptaphylla, both species belonging to the Rutaceae family, have shown important results with respect to their chemistry. These species have secondary metabolites (coumarins and alkaloids mainly), which act as

* carlos.coy@unimilitar.edu.co 
chemotaxonomic markers. This paper presents, for the first time, the phytochemical work and isolated metabolites in these species: four coumarins: bergapten, xanthyletin, xanthotoxin, 3-isoprenyl-4-methoxy-coumarin; four alkaloids: skinmianine, kokusaginine, dictamnine, and 1-methyl-2methoxy-4-quinolone; two lignans: sesamin and mesodihydroguaiaretic acid; two sterols: $\beta$-sitosterol, stigmasterol; and a pentacyclic triterpene: lupeol that have been isolated from leaves of E. alata. Five alkaloids 7-methoxy-2,2-dimethyl-2,6-dihidro-piran[3,2,c] quinolin-5-one, flindersiamine, skinmianine, kokusaginine and dictamnine that have been isolated from leaves of $R$. heptaphylla. Moreover, it presents a new method by obtaining quinolone alkaloid analogue precursors trough the condensations of Mannich adduct vinylogous and aldehydes followed by radical cyclization to obtain products with high regio- and stereoselectivity.

Palabras Clave: Raputia, rutácea, esenbeckia, alcaloides quinolínicos

Keywords: Raputia, Rutaceae, Esenbeckia, quinolinic alkaloids

\section{Introducción}

La familia Rutaceae está constituida por cerca de 150 géneros y 1600 especies, muchas de las cuales han sido reportadas por su amplio uso en medicina folklórica como analgésico, antipirético, ansiolítico y antiinflamatorio, entre otras aplicaciones y para el tratamiento de algunas enfermedades y afecciones tales como malaria, reumatismo, úlceras digestivas, gastritis, resfriado, fiebre y diarrea [1].

En Colombia la especie Esenbeckia alata se encuentra en los departamentos del Valle y Sucre, Esenbeckia amazonia koastra en Boyacá, Esenbeckia panamensis en el Chocó, Esenbeckia litoralis en Bolívar y Esenbeckia pentaphylla en el Magdalena. En el país el uso que se le da principalmente es como fuente maderable. En América especies como Esenbeckia yaxhoob y Esenbeckia leiocarpa tienen una etnobotánica interesante y son usadas para curar dolencias estomacales, diarreas y disentería [2]. Del género Esenbeckia se han aislado algunos alcaloides, cumarinas y quinolonas característicos de este género [2].

El género Raputia no ha sido muy estudiado, pues existen reportes de investigaciones hechas en Indonesia y Brasil donde se han aislado flavonoides glicosidados [3] y alcaloides indólicos [4] respectivamente. Muchas especies de esta familia, se han estudiado con el fin de confirmar la actividad biológica que sustenta su uso en la medicina tradicional, y así determinar el tipo de metabolito que presenta la acción farmacológica observada, y establecer cómo actúa el producto activo dentro del organismo.

Las investigaciones sobre la actividad biológica in vitro, se han centrado principalmente en los alcaloides y las cumarinas, debido a su gran importancia en el campo farmacológico [5].

Estos metabolitos secundarios presentan un amplio rango de actividades biológicas como son, antitumoral [6], astringente [6], analgésica [7], de antiagregación plaquetaria [8], antimicrobiana [9], leishmanicida [10], citotóxica [11], anticoagulante [12] y antimalárica [13].

Los alcaloides furoquinolínicos comunes en el género Esenbeckia, han sido estudiados en otras especies durante los últimos años, arrojando resultados interesantes; un ejemplo es el estudio realizado al extracto de la corteza de Melicope semicarpifolia (Rutaceae), el cual posee actividad de antiagregación plaquetaria debido a la cantidad de alcaloides 
furoquinolínicos que posee (kokusaginina, skinmianina, evolitrina, haplopina). En el año 2006 se pudo observar la marcada actividad antifúngica de alcaloides como kokusaginina, maculina, dictamnina contra Leucoagaricus gonylophorus [14].

La haplopina, skinmianina, $\gamma$-fagarina y otros alcaloides furoquinolínicos que están en el género Haplophyllum y Esenbeckia, se caracterizan por poseer actividad estrogénica, comparada con derivados de dihidrofuroquinolinas y alcaloides quinolónicos [14].

La síntesis orgánica en la actualidad juega también un papel importante en la producción de nuevos compuestos que puedan presentar aplicaciones terapéuticas basadas en su gran mayoría en los metabolitos provenientes de fuentes vegetales, principalmente con núcleos nitrogenados. Por consiguiente, además del trabajo fitoquímico, se presenta la síntesis de los precursores y análogos de alcaloides quinolínicos, por medio de la condensación de aductos de Mannich vinílogos 1 y aldehídos 2 para formar enaminas cíclicas 3. (Figura 1). Esta reacción ha sido útil en la preparación de piperidinas polisustituidas vía reacciones de hidrogenación, adición nucleofílica y acoplamientos de tipo reductivo [15].

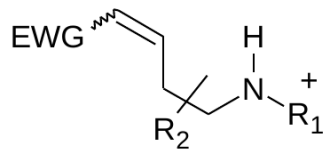

1

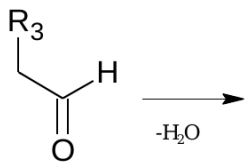

2<smiles>[R14]CC1([R2])CC([R3])=CN([R1])C1</smiles>

3

Figura 1. Formación de tetrahidropiridinas a partir de aminas y aldehídos.

Estas enaminas sustituidas 4, comúnmente llamadas tetrahidropiridinas, pueden ser, en principio, precursores en la formación de sistemas policíclicos $\mathbf{6}$ y $\mathbf{7}$, vía ciclación radicalaria [16] a través del intermediario 5. Los compuestos cíclicos 6 y 7 (Figura 2 ), resultan interesantes porque este tipo de esqueletos presentan un gran número de compuestos biológicamente activos [17]. La ciclación radicalaria intramolecular en enaminas con sustituyentes de tipo alquilo y enamidas es bien conocida [18], sin embargo para el caso de enaminas cíclicas como 4 solo existen reportes empleando sulfonilaminas [19] y derivados benzocarbazólicos e indólicos [20] [21] que se han caracterizado por ser poco regioselectivas y además, en cada caso se obtienen mezclas de los productos de ciclación 5-exo-trig y 6-exo-trig, formando anillos de cinco y seis miembros, respectivamente. Por lo tanto, vale la pena mencionar que este trabajo es el primer ejemplo de ciclaciones radicalarias para la formación de compuestos de tipo 6 que no envuelven derivados indólicos.

\section{Parte experimental}

\subsection{Material vegetal}

La muestra de E. alata (hojas, corteza y madera), fue recolectada en Colosó, Sucre por el biólogo Olimpo García y el doctor Luis Enrique Cuca Súarez profesor titular 


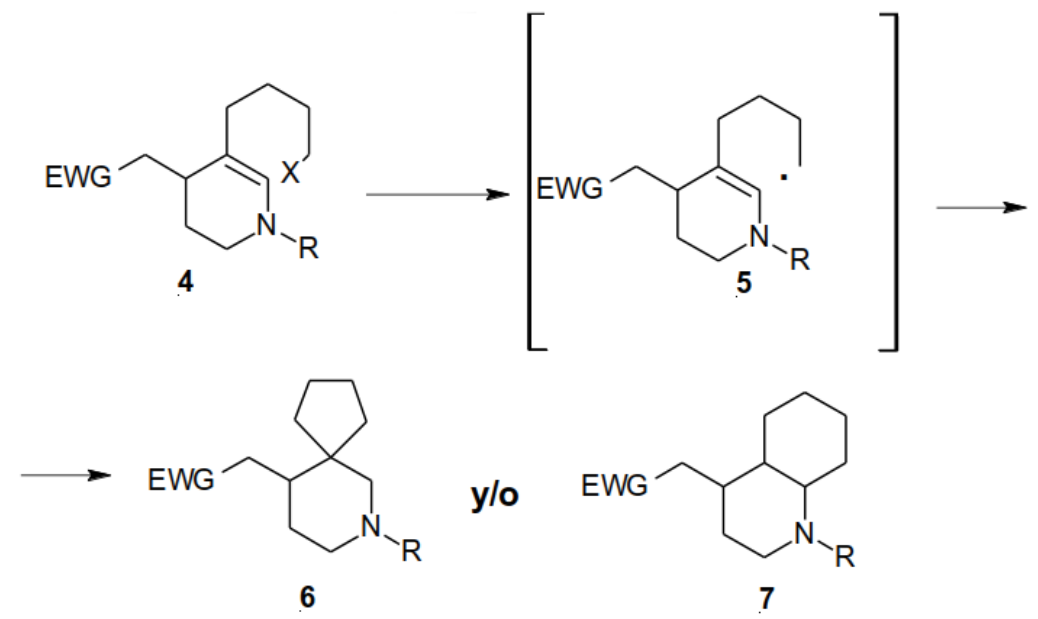

Figura 2. Síntesis de 6 y 7.

de la Universidad Nacional. Un ejemplar reposa en el Herbario Nacional de Colombia, Instituto de Ciencias Naturales de la Universidad Nacional de Colombia bajo el COL. 481090. La muestra de R. heptaphylla hojas, corteza y madera fue recolectada en Albán, municipio de Cundinamarca, por el biólogo Zaleth Cordero, el doctor Luis Enrique Cuca Suárez profesor titular de la Universidad Nacional, Carlos Andrés Coy Barrera y Oscar Javier Patiño, estudiantes de Doctorado en Ciencias - Química. Un ejemplar reposa en el Herbario Nacional de Colombia, Instituto de Ciencias Naturales de la Universidad Nacional de Colombia bajo el COL. $\underline{511102}$.

\subsection{Aislamiento, purificación de metabolitos aislados de las especies E. alata y $R$. heptaphylla}

El material vegetal de hojas de E. alata $(750 \mathrm{~g})$, fue sometido a extracción por percolación con EtOH al $96 \%$. A este extracto se le realizó partición por Soxhlet con EdP (22 g), $\mathrm{CHCl}_{3}(42 \mathrm{~g})$ y AcOEt $(35 \mathrm{~g})$. El extracto de EdP fue fraccionado por CC usando como mezclas de elución Tol: AcOEt en polaridad creciente (9:1-1:1). Las fracciones obtenidas fueron purificadas usando CC repetitiva, y CCDP utilizando como eluyentes mezclas de $\mathrm{CHCl}_{3}$ : $\mathrm{MeOH}$ y n-hexano: AcOEt con gradiente de elución. De este extracto se obtuvieron bergapteno 8 (17 mg), xantotoxina 9 (20 mg), xantiletina 10 (15 mg), skinmianina 11 (16 mg), lupeol 12 (1 4mg), sesamina 13 (14 mg). El extracto de AcOEt fue fraccionado por CC utilizando Tolueno: AcOEt como mezcla de elución. La purificación de las sustancias fue realizada por CC repetitiva y CCDP, eluyendo con $\mathrm{CHCl}_{3}$ : MeOH y n-hexano: AcOEt en polaridad creciente (9:1-1:1), obteniéndose, kokusaginina 15 (10 mg), dictamnina 16 (19 mg), 1-metil-2-metoxi-4-quinolona 17 (8mg), 3-isoprenil-4-metoxi-cumarina 18 (11 mg). El material vegetal de hojas de $R$. heptaphylla, seco y molido (1700 g), fue sometido a percolación con etanol al $96 \%$, y se obtuvieron $80 \mathrm{~g}$ de extracto; $1 \mathrm{~g}$ se tomó para el análisis fitoquímico preliminar y el resto para el fraccionamiento y purificación. Según pruebas de coloración (Draggendorf), 
se determinó la presencia de alcaloides en la muestra; por ello se realizó una extracción típica ácido-base para este tipo de sustancias a $40 \mathrm{~g}$ del extracto etanólico original, de lo cual se obtuvo $1.7 \mathrm{~g}$ de extracto clorofórmico de alcaloide que fue fraccionado por $\mathrm{CC}$ utilizando mezclas de elución $\mathrm{CHCl}_{3}$ : $\mathrm{ACN}$ y $\mathrm{CHCl}_{3}$ : AcOEt en polaridad creciente. Los metabolitos aislados se purificaron por CC repetitiva y CCDP, para obtener los compuestos skinmianina 11 (25 mg), kokusaginina 15 (44 mg), 16 dictamnina (29 mg), el alcaloide piranoquinolínico 7-metoxi-2,2-dimetil-2,6-dihidro-pirano[3,2,c] quinolin-5ona $\mathbf{1 9}(6,5 \mathrm{mg})$ y flindersiamina $(14,5 \mathrm{mg}) \mathbf{2 0}$.

\section{Resultados y discusión}

\subsection{Aislamiento, purificación y elucidación estructural de metabolitos aislados de las especies E. alata y R. heptaphylla}

Del extracto de EdP de hojas de E. alata se obtuvieron dos furocumarinas bergapteno [22] 8, xantotoxina [22] 9, una piranocumarina, xantiletina [22] 10 un alcaloide furoquinolínico skinmianina [22] 11, un triterpeno, lupeol [22] 12, y dos lignanos (-)-sesamina [22] 13 y ácido meso-dihidroguaiarético [23] 14.
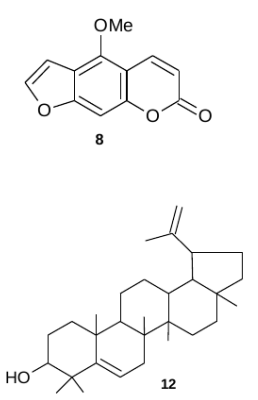
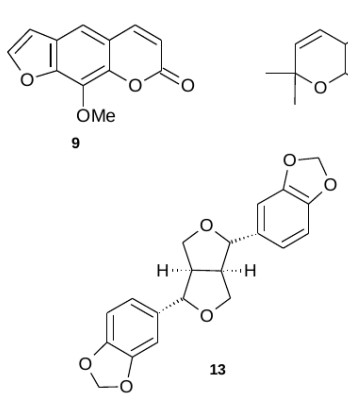

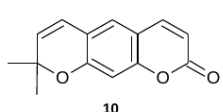

10
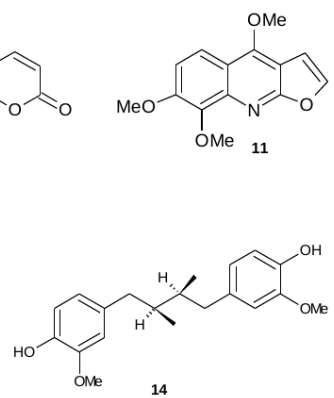

Figura 3. Metabolitos aislados del extracto EdP hojas de E. alata.

Del extracto de AcOEt de hojas de E. alata se aislaron dos alcaloides furoquinolínicos, kokusaginina [24] 15 y dictamnina [24] 16, un alcaloide quinolónico, 1-metil-2-metoxi-4quinolona24 17, una cumarina prenilada, 3-isoprenil-4-metoxi-cumarina [22] 18. Además $\beta$-sitosterol [25] y estigmasterol [25].

Al extracto etanólico de hojas de la especie $R$. heptaphylla, obtenido por percolación, se le realizó extracción de alcaloides utilizando el método ácido-base [26], ya que según el análisis fitoquímico preliminar [27] se encontró gran porcentaje de este tipo de metabolitos. De dicha extracción se obtuvieron $1,7 \mathrm{~g}$ de extracto clorofórmico de alcaloides, el cual fue fraccionado por CC utilizando como mezclas de elución $\mathrm{CHCl}_{3}$ : ACN y $\mathrm{CHCl}_{3}$ : AcOEt en polaridad creciente, los metabolitos aislados se purificaron por CC repetitiva, y CCDP, para obtener los compuestos 11, 15, 16 que ya habían sido aislados de la especie E. alata. El alcaloide piranoquinolínico 7-metoxi-2,2-dimetil-2,6dihidro-pirano $[3,2, c]$ quinolin-5-ona 19, aislado anteriormente de especies de la familia 
<smiles>COc1cc2nc3occc3c(OC)c2cc1OC</smiles>

15<smiles>COc1cc(=O)c2ccccc2n1C</smiles><smiles>COc1c2ccccc2nc2occc12</smiles>

16

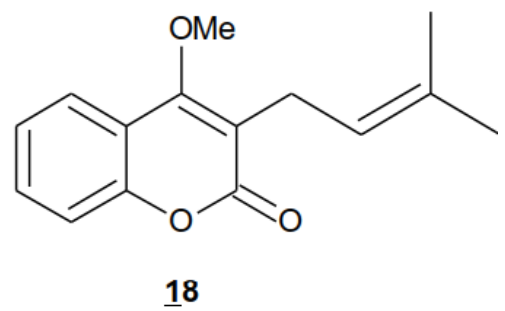

Figura 4. Metabolitos aislados del extracto AcOEt hojas de E. alata.

Rutaceae, específicamente del género Haplophyllum [28], y la flindersiamina 20, aislada anteriormente de Raullinoa echinata [29] (Rutaceae), sin embargo resultan interesantes porque no existen reportes de este tipo de alcaloides en el género Raputia.

El compuesto 19 es un aceite amarillo, el espectro IR ( NaCl), muestra en $2980 \mathrm{~cm}^{-1}$ estiramiento $\mathrm{C}-\mathrm{H}$ alifático, en $1720 \mathrm{~cm}^{-1}$ estiramiento $\mathrm{C}=\mathrm{O}$ de carbonilo característico de lactona, confirmado por el estiramiento $\mathrm{C}-\mathrm{O}$ de lactona en $1160 \mathrm{~cm}^{-1}$, el espectro de RMN - ${ }^{1} \mathrm{H}$ muestra señales aromáticas en $\delta 7.60(\mathrm{dd}, J=7.9,1.47 \mathrm{~Hz}, 1 \mathrm{H}), 7.13$ (dd, $J=7.9 \mathrm{~Hz}, 1 \mathrm{H}), 7.05(\mathrm{dd}, J=7.9,1.4 \mathrm{~Hz}, 1 \mathrm{H})$, que corresponde a un sistema de tres protones aromáticos $\mathrm{ABC}$, en un anillo trisustituido, 6.75 (dd, $J=9.8 \mathrm{~Hz}$, $1 \mathrm{H}), 5.52(\mathrm{dd}, J=9.8 \mathrm{~Hz}, 1 \mathrm{H})$, protones vinílicos, $3.92(\mathrm{~s}, 3 \mathrm{H}, \mathrm{O}-\mathrm{Me}), 3.88(\mathrm{~s}, 3 \mathrm{H}$, $\mathrm{N}-\mathrm{Me}), 1.50(\mathrm{~s}, 6 \mathrm{H})$, metilos geminales. La comparación entre el espectro RMN $-{ }^{13} \mathrm{C}$ y DEPT 135, permite establecer la presencia de 7 carbonos cuaternarios en $\delta 78.7(\mathrm{C}$ unido a oxígeno), 106.0 (C), 118.3 (C), 131.1 (C), 148.6 (C), 154.9 (C), 162.1 (C=O), 5 metinos en $\delta 114.3(\mathrm{CH}), 115.6(\mathrm{CH}), 118.0(\mathrm{CH}), 122.2(\mathrm{CH}), 126.6(\mathrm{CH}) 2$ metilos equivalentes en $\delta 28.2\left(2 \mathrm{CH}_{3}\right)$, y en $\delta 35.1\left(\mathrm{CH}_{3}-\mathrm{N}\right), 56.7\left(\mathrm{CH}_{3}-\mathrm{O}\right)$. La estructura de la molécula fue determinada de acuerdo a los datos espectroscópicos y confirmados por espectros bidimensionales HMQC y HMBC. También se determinó por el método de la comparación con los datos reportados en la literatura [28], y finalmente se aplicó el mismo método con los demás compuestos puros aislados de E. alata y $R$. heptaphylla.

\subsection{Síntesis de precursores y análogos quinolínicos}

Se llevó a cabo inicialmente la protección del grupo amino de la bencilamina $\mathbf{2 1}$ con incorporación de un grupo tert-butoxicarbonilo, obteniéndose el correspondiente derivado Boc protegido $\mathbf{2 2}$ con un $90 \%$ de rendimiento. A continuación, se efectuó la alquilación del $N$ amídico por desprotonación con $\mathrm{n}-\mathrm{BuLi}$, a baja temperatura y posterior adición de clorometil metil eter; así se obtuvo el correspondiente N-metoximetilderivado $\mathbf{2 3}$ con un rendimiento del $88 \%$. (Figura 6). 


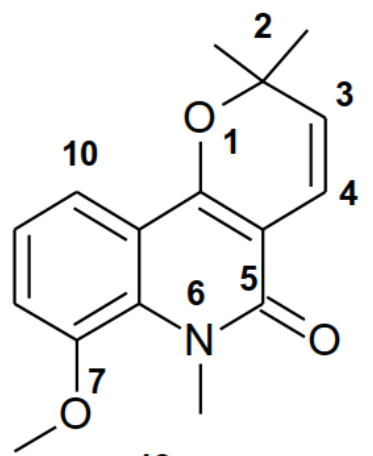

19<smiles></smiles>

$\underline{20}$

Figura 5. Metabolitos aislados de R. heptaphylla<smiles>NCc1ccccc1</smiles>

21

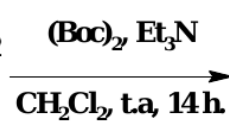

$90 \%$<smiles>CC(C)(C)OC(=O)NCc1ccccc1</smiles>

22

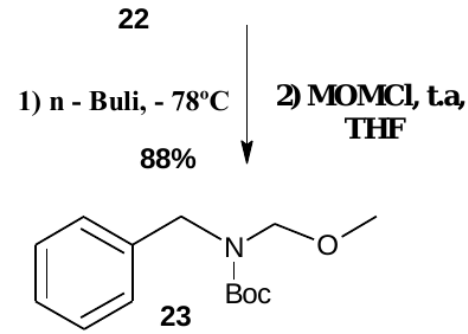

Figura 6. Síntesis de $N$-Metoximetil-derivado 23.

El $N$-metoximetilcarbamato $\mathbf{2 3}$ se usó como sustrato para una reacción de Mannich viníloga a $-78{ }^{\circ} \mathrm{C}$, con 2 -sililoxifurano, usando TMSOTf como agente activante en las condiciones descritas por Aurrecoechea et al. [15]. De este modo, se obtuvo una mezcla de la amina $\mathbf{2 4}$ junto con su regioisómero $\mathbf{2 4 a}$ con un $95 \%$ de rendimiento global y una relación 4:1. Para completar la síntesis de la amina 25, el carbamato $\mathbf{2 4}$ [15] que se purificó mediante sucesivas precipitaciones con hexano: Eter etílico 4:1 a $0{ }^{\circ} \mathrm{C}$, se sometió a hidrólisis ácida con ácido trifluoroacético (TFA), dando lugar a la amina deseada. Esta amina se hizo reaccionar con el aldehído $\mathbf{2 7}$ formado a partir de la oxidación de 6-bromohexanol 26, con $\mathrm{PCC}$ en $\mathrm{CH}_{2} \mathrm{Cl}_{2}$, y a $0{ }^{\circ} \mathrm{C}$, siguiendo el procedimiento descrito por Hon et al. [30]. La reacción equimolar entre la amina 25 y el aldehído 27 en $\mathrm{CH}_{2} \mathrm{Cl}_{2}$ y en presencia de tamiz molecular de $4 \AA$, induce a la formación de la tetrahidropiridina 28. (Figura 7). 


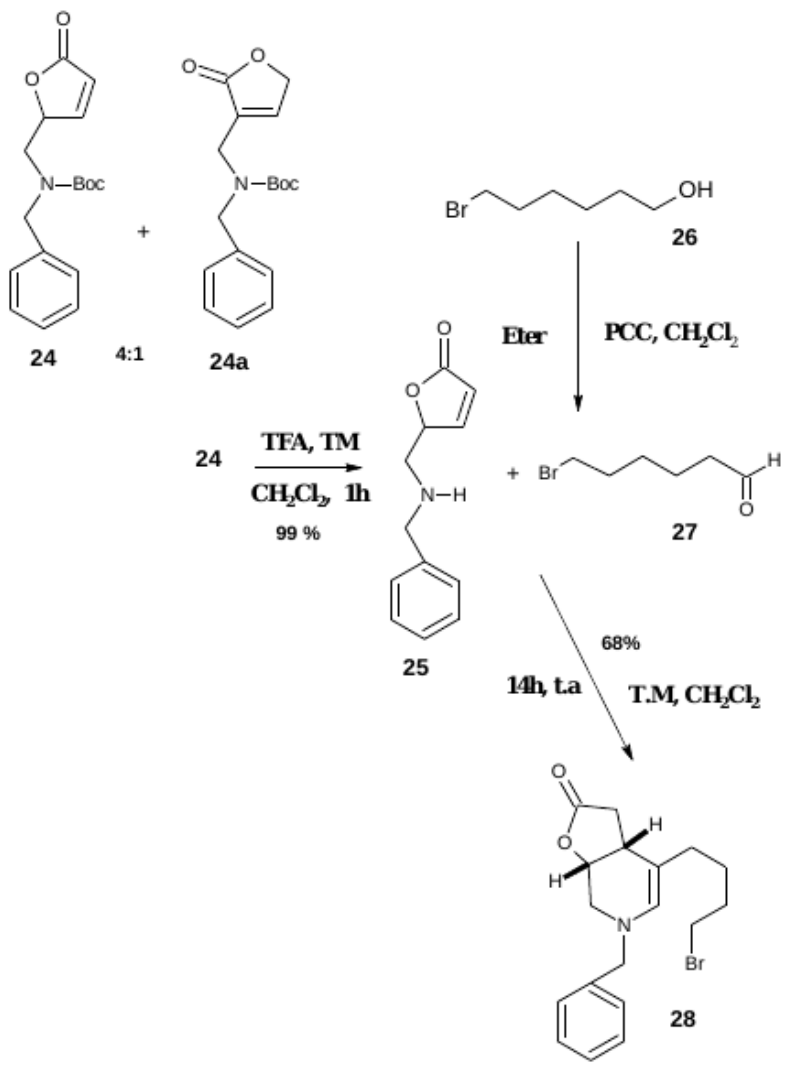

Figura 7. Síntesis de $\left(1 \mathrm{R}^{*}, 5 \mathrm{R}^{*}\right)$-8-Bencil-6-(4-bromobutil)-2-oxa-8-azabiciclo [3.4.0]non-6-en-3-ona, Tetrahidropiridina 28.

A partir de la tetrahidropiridina $\mathbf{2 8}$ se hizo la ciclación radicalaria. Esta reacción se realizó utilizando AIBN como iniciador en la formación de radicales libres, a una temperatura de $100{ }^{\circ} \mathrm{C}$ benceno y $\mathrm{TMS}_{3} \mathrm{SiH}$, teniendo en cuenta las condiciones descritas en la bibliografía para otro tipo de sustratos [31], en donde se observó que la formación del producto espiránico 29, es de $45 \%$ (Figura 8).

A partir de la amina $\mathbf{2 5}$ y del 6-bromo-4,4'-dimetilhept-6-enal [32], se obtuvo la tetrahidropiridina $\mathbf{3 0}$ con un porcentaje de rendimiento del $69 \%$, la posterior ciclación radicalaria bajo las mismas condiciones anteriores produjo la formación de dos isómeros 31 y 32 en relación 4:1, mediante CC flash, se logró purificar el compuesto 31, que sirvió para la determinación de la proporción de la mezcla y posterior elucidación del compuesto 32 [33] (Figura 9). La estereoquímica del compuesto 31 se estableció a partir del análisis de los experimentos NOE. 
<smiles>O=C1C[C@H]2C(CCCCBr)=CN(Cc3ccccc3)C[C@H]2O1</smiles>

28

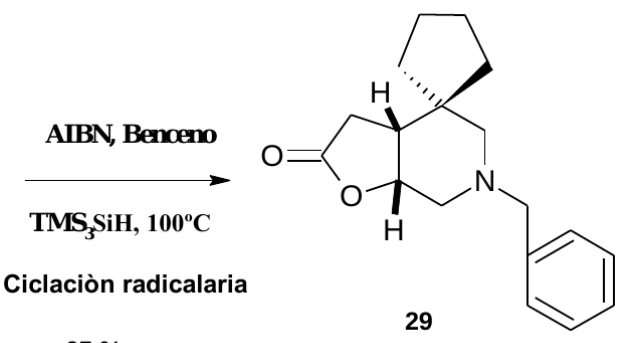

$65 \%$

Figura 8. (1R $\left.\mathrm{R}^{*}, 5 \mathrm{R}^{*}\right)$-8-bencil-2-oxa-8-azabiciclo-[3.4.0]-non-6-en-3-ona-6-espirociclopentano 29.<smiles>CC1(C)CC(Br)=C2OC(=O)CCC2C2CCN(Cc3ccccc3)CC21</smiles>

30

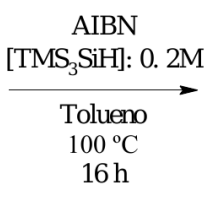

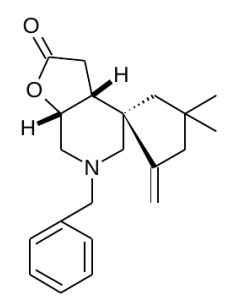

31

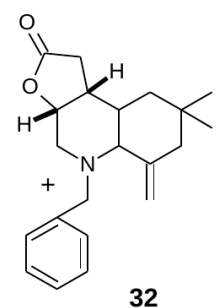

32

Figura 9. (1R $\left.\mathrm{R}^{*}, 5 \mathrm{R}^{*}, 6 \mathrm{~S}^{*}\right)$-8-bencil-2-oxa-8-azabiciclo[3.4.0]nonan-3-ona-6-espiro,4'(1',1'-dimetil-3'-metilénciclopentano)] 31 y $\left(3 \mathrm{aR}^{*}, \quad 9 \mathrm{aR}^{*}\right)$-5-bencil-8,8-dimetil-6metiléndecahidrofuro [2,3-c] quinolin-2-ona $\mathbf{3 2}$.

\subsection{Síntesis de $\left(1 R^{*}, 5 R^{*}\right)-8-B e n c i l-6-(4 '-b r o m o b u t i l)-2-o x a-8-$ azabiciclo [3.4.0]-non-6-en-3-ona 28}

Sobre una disolución de la amina 25 (340 mg, 1,67 mmol) y el aldehido 27 (299 mg, $1,66 \mathrm{mmol})$ en diclorometano $(21 \mathrm{~mL})$, se adicionó tamiz molecular de $4 \AA(3,44 \mathrm{~g})$ y la suspensión resultante se agitó a temperatura ambiente durante 14 horas. Se filtró el residuo sólido sobre Celita y se lavó con diclorometano $(3 \times 15 \mathrm{~mL})$. El aceite de color marrón oscuro resultante de la evaporación de los disolventes se purificó por cromatografía flash (gel de sílice, saturada con $\mathrm{Et}_{3} \mathrm{~N}$, hexano:AcOEt:Et ${ }_{3} \mathrm{~N}=85: 13: 2$, obteniéndose $\mathbf{2 8}$ (413 mg, $68 \%$ ) como un aceite amarillo claro, que se descompone con rapidez a temperatura ambiente: $\mathrm{RMN}^{1}{ }^{1} \mathrm{H}\left(300 \mathrm{MHz}, \mathrm{CDCl}_{3}\right) \delta 1.42-2.01(\mathrm{~m}, 6 \mathrm{H}), 2.36$ $(\mathrm{dd}, J=17.4,5.9 \mathrm{~Hz}, 1 \mathrm{H}), 2.75(\mathrm{dd}, J=17.4,8.6, \mathrm{~Hz}, 1 \mathrm{H}), 2.91-3.01(\mathrm{~m}, 3 \mathrm{H}), 3.42$ $(\mathrm{td}, J=6.7,0.9 \mathrm{~Hz}, 2 \mathrm{H}), 3.98$ y $4.03\left(\mathrm{c} \mathrm{AB}, J=14,7 \mathrm{~Hz}, 2 \mathrm{H}, \mathrm{Ph}-\mathrm{CH}_{2}\right), 4.75$ (ddd, $J$ $=6.9,5.4,4.5 \mathrm{~Hz} 1 \mathrm{H}, \mathrm{H}-1), 5.95(\mathrm{~s}, 1 \mathrm{H}, \mathrm{H}-7), 7.24-7.37(\mathrm{~m}, 5 \mathrm{H}, \mathrm{Ar}) ; \mathrm{RMN}^{13} \mathrm{C}(75$ $\left.\mathrm{MHz}, \mathrm{CDCl}_{3}\right) \delta 26.6\left(\mathrm{CH}_{2}\right), 31.6\left(\mathrm{CH}_{2}\right), 32.1\left(\mathrm{CH}_{2}\right), 33.7\left(\mathrm{CH}_{2}\right), 34.3(\mathrm{CH}, \mathrm{C} 5), 34.8$ $\left(\mathrm{CH}_{2}\right), 47.9\left(\mathrm{CH}_{2}\right), 59.2\left(\mathrm{CH}_{2}\right) 77.5(\mathrm{CH}, \mathrm{C} 1), 107.0(\mathrm{C}, \mathrm{C} 6), 127.5(\mathrm{CH}), 128.1(\mathrm{CH})$, $128.1(\mathrm{CH}), 128.6(\mathrm{CH}), 133.4(\mathrm{CH}), 137.4(\mathrm{C}), 176.5(\mathrm{C}=\mathrm{O})$; IR (Neto) $2932(\mathrm{~m},=\mathrm{CH})$ 1772 (f, C=O), 1660 (m, C=C), 1161 (m, C-O) cm ${ }^{-1}$; EM (IE) m/z (\%) (365) (4), (364) (1), (242) (95), (238) (13), (91) (base), EMAR calculado para $\mathrm{C}_{18} \mathrm{H}_{22} \mathrm{O}_{2} \mathrm{Br}^{79} \mathrm{~N}$ 
363.0830, encontrado 363.0834. Calculado para $\mathrm{C}_{18} \mathrm{H}_{22} \mathrm{O}_{2} \mathrm{Br}^{81} \mathrm{~N}$ : 365.0804, encontrado: 365.0813 .

\subsection{Síntesis de $\left(1 R^{*}, 5 R^{*}\right)$-8-bencil-2-oxa-8-azabiciclo[3.4.0]non-6- en-3-ona-6-espirociclopentano 29}

Se calentó una disolución de 28 (349 mg, $0.964 \mathrm{mmol})$, AIBN (158 mg, $0.2892 \mathrm{mmol}$ ) y $\mathrm{TMS}_{3} \mathrm{SiH}\left(548 \mathrm{mg}, 1.928 \mathrm{mmol}\right.$ ) en tolueno $(9.64 \mathrm{~mL})$ a $100^{\circ} \mathrm{C}$ (temperatura del baño de aceite) durante 16 horas bajo Argón, tras enfriar, la mezcla se diluyó con AcOEt (15 $\mathrm{mL})$ seguido por la adición de $\mathrm{HCl} 1 \mathrm{M}(20 \mathrm{~mL})$. Se separó la fase orgánica de la fase acuosa y ésta se extrajo con $\mathrm{HCl}(3 \times 20 \mathrm{~mL})$, los extractos ácidos se basificaron con $\mathrm{NaHCO}_{3}(2 \mathrm{M})$ hasta $\mathrm{pH} 8$ (aproximadamente $120 \mathrm{~mL}$ ), se extrajeron con $\mathrm{CH}_{2} \mathrm{Cl}_{2}(3$ $\times 25 \mathrm{~mL}$ ) y los extractos orgánicos se lavaron con salmuera y se secaron con $\mathrm{Na}_{2} \mathrm{SO}_{4}$ anhidro. Tras la evaporación, el residuo (aceite marrón oscuro, 277,7 mg) se purificó por CC flash (gel de sílice saturada con $\mathrm{Et}_{3} \mathrm{~N}$, Hex:AcOEt:Et ${ }_{3} \mathrm{~N}, 90: 7: 3$ ), obteniéndose 29 como un sólido blanco (179 mg, $65 \%)$ : p.f: $76-78{ }^{\circ} \mathrm{C}$; RMN- ${ }^{1} \mathrm{H}\left(300 \mathrm{MHz}, \mathrm{CDCl}_{3}\right) \delta$ $1.28-1.57(\mathrm{~m}, 7 \mathrm{H}), 1.78-1.86(\mathrm{~m}, 1 \mathrm{H}), 2.05(\mathrm{dt}, J=11.5,8.9 \mathrm{~Hz}, 1 \mathrm{H}), 2.21-2.50(\mathrm{~m}, 4 \mathrm{H})$, 3.01 (ddd, $J=11.3,5.7,1.5 \mathrm{~Hz}, 1 \mathrm{H}$ ), 3.43 y 3.51 (c AB, $J=13.3 \mathrm{~Hz}, 2 \mathrm{H}, \mathrm{Ph}_{-} \mathrm{CH}_{2}$ ), $4.60(\mathrm{dt}, J=9.3,6.3 \mathrm{~Hz}, 1 \mathrm{H}), 7.19-7.30(\mathrm{~m}, 5 \mathrm{H}, \mathrm{Ar}) ; \mathrm{RMN}-{ }^{13} \mathrm{C}\left(75 \mathrm{MHz}, \mathrm{CDCl}_{3}\right)$ $\delta 23.9\left(\mathrm{CH}_{2}\right), 24.2\left(\mathrm{CH}_{2}\right), 29.8\left(\mathrm{CH}_{2}\right), 35.4\left(\mathrm{CH}_{2}\right), 37.9\left(\mathrm{CH}_{2}\right), 43.7(\mathrm{CH}), 44.2(\mathrm{C})$, $54.8\left(\mathrm{CH}_{2}\right), 57.8\left(\mathrm{CH}_{2}\right), 62.5\left(\mathrm{CH}_{2}\right), 76.4(\mathrm{CH}), 127.2(\mathrm{CH}), 128.3(\mathrm{CH}), 128.7(\mathrm{CH})$, $138.0(\mathrm{C}), 176.7(\mathrm{C}=\mathrm{O})$; IR (Neto) $1772(\mathrm{f}, \mathrm{C}=\mathrm{O}), 1155(\mathrm{~m}, \mathrm{C}-\mathrm{O}) \mathrm{cm}^{-1} ; \mathrm{EM}(\mathrm{IE}) \mathrm{m} / \mathrm{z}$ (\%) (285) (17), (194) (39) (69) (base). EMAR: calculado para $\mathrm{C}_{18} \mathrm{H}_{23} \mathrm{NO}_{2} 285.1729$, encontrado: 285.1721.

\subsection{Síntesis de $\left(1 R^{*}, 5 R^{*}\right)-8$-Bencil-6-(2,2-dimetil-4-bromopent-4- enil)-2-oxa-8-azabiciclo [3.4.0] non-6-en-3-ona 30}

Sobre una disolución de la amina $24(190 \mathrm{mg}, 0.94 \mathrm{mmol})$ y el aldehido 6-bromo-4,4dimetilhept-6-enal [32] (205 mg, 0,94 mmol) en diclorometano (13 mL), se adicionó tamiz molecular pulverizado de $4 \AA(2,00 \mathrm{~g})$ y la suspensión resultante se agitó a temperatura ambiente durante 17 horas. Se filtró el residuo sólido sobre Celita y se lavó con diclorometano $(3 \times 15 \mathrm{~mL})$. El aceite resultante de la evaporación de los disolventes se purificó por cromatografía flash (gel de sílice saturada con $\mathrm{Et}_{3} \mathrm{~N}$, hexano:AcOEt:Et ${ }_{3} \mathrm{~N}$ $=90: 7: 3$, obteniéndose 30 como un sólido blanco $(185 \mathrm{mg}, 49 \%)$ : p.f $=46-48{ }^{\circ} \mathrm{C}$; $\mathrm{RMN}^{1} \mathrm{H}\left(300 \mathrm{MHz}, \mathrm{CDCl}_{3}\right) \delta 0.98$ y $0.99(2 \mathrm{~s}, 6 \mathrm{H}), 1.91$ y $1.86(\mathrm{c} \mathrm{AB}, J=14,7 \mathrm{~Hz}$, $2 \mathrm{H}), 2.36-2.48(\mathrm{~m}, 3 \mathrm{H}), 2.76(\mathrm{dd}, J=17.1,8.4 \mathrm{~Hz}, 1 \mathrm{H}), 2.88(\mathrm{dd}, J=12.3,2.5 \mathrm{~Hz}$, $1 \mathrm{H}), 3.07$ (dd, $J=12.2,5.1 \mathrm{~Hz}$, solapado con señales de otro $\mathrm{H}$, total $2 \mathrm{H}), 4.01$ y 4.08 $\left(\mathrm{c} \mathrm{AB}, J=14,7 \mathrm{~Hz}, 2 \mathrm{H}, \mathrm{Ph}-\mathrm{CH}_{2}\right), 4.73-4.77(\mathrm{~m}, 1 \mathrm{H}, \mathrm{H}-1), 5.51(\mathrm{~s}, 1 \mathrm{H}),, 5.56(\mathrm{~d}, J=$ $1.1 \mathrm{~Hz}, 1 \mathrm{H}), 5.97(\mathrm{~s}, 1 \mathrm{H}), 7.25-7.37(\mathrm{~m}, 5 \mathrm{H}, \mathrm{Ar})$; RMN- ${ }^{13} \mathrm{C}\left(75 \mathrm{MHz}, \mathrm{CDCl}_{3}\right) \delta 26.9$ $\left(\mathrm{CH}_{3}\right), 27.5\left(\mathrm{CH}_{3}\right), 35.1\left(\mathrm{CH}_{2}\right), 35.9(\mathrm{C}) 36.2(\mathrm{CH}), 44.9\left(\mathrm{CH}_{2}\right), 47.6\left(\mathrm{CH}_{2}\right), 53.3\left(\mathrm{CH}_{2}\right)$, $59.2\left(\mathrm{CH}_{2}\right) 77.9(\mathrm{CH}), 103.7(\mathrm{C}), 120.5\left(\mathrm{CH}_{2}\right), 127.5(\mathrm{CH}), 128.0(\mathrm{CH}), 128.6(\mathrm{CH})$, $130.3(\mathrm{C}), 136.8(\mathrm{CH}), 137.4(\mathrm{C}), 176.7(\mathrm{C}=\mathrm{O})$. IR (Neat) $1772(\mathrm{~s}, \mathrm{C}=\mathrm{O}), 1649(\mathrm{~m}$, $\mathrm{C}=\mathrm{C}) \mathrm{cm}^{-1}$; EM (IE) m/z, 405 (M, 0.44), 403 (M, 0.38), 324 (9), 242 (99), 91 (base). EMAR: calculado para $\mathrm{C}_{21} \mathrm{H}_{26}{ }^{79} \mathrm{BrNO}_{2}$ 403.1147, encontrado: 403.1141, calculado para $\mathrm{C}_{21} \mathrm{H}_{26}{ }^{81} \mathrm{BrNO}_{2}$ 405.1126, encontrado: 405.1136. 


\subsection{Síntesis $\left(1 \mathrm{R}^{*}, 5 \mathrm{R}^{*}, 6 \mathrm{~S}^{*}\right)$-8-bencil-2-oxa-8-azabiciclo[3.4.0]nonan- 3-ona-6-espiro , 4'-(1',1'-dimetil-3'-metilénciclopentano)] 31 y $\left(3 \mathrm{aR}^{*}, 9 \mathrm{aR}^{*}\right)$-5-bencil-8,8-dimetil-6-metiléndecahidrofuro [2,3-c]quinolin-2-ona 32}

El compuesto 30 (159 mg, 0,394 mmol) fue mezclado con AIBN (194 mg, $0.118 \mathrm{mmol}$ ) y $\mathrm{TMS}_{3} \mathrm{SiH}$ (196 mg, 0,690 mmol), bajo atmósfera de Argón y repetidas purgas vacíoargón, se adicionó tolueno $(3.94 \mathrm{~mL})$ y la solución resultante fue introducida en un baño de aceite con agitación constante a $100^{\circ} \mathrm{C}$ por 16 horas, se elaboró la reacción al solubilizar el crudo en AcOEt (10 mL) seguido por la adición de HCl 1M (10 mL), se separó la fase orgánica ácida de la fase acuosa y a esta última se le realizaron extracciones con $\mathrm{HCl} 1 \mathrm{M}(3 \times 10 \mathrm{~mL})$. Luego se reunieron las fases ácidas y se neutralizaron con $\mathrm{NaHCO}_{3}(80 \mathrm{~mL})$ hasta $\mathrm{pH}$, se hizo extracción con $\mathrm{CH}_{2} \mathrm{Cl}_{2}(3 \times 15 \mathrm{~mL})$, lavado con salmuera y adición de $\mathrm{Na}_{2} \mathrm{SO}_{4}$ anhidro, el residuo obtenido fue filtrado y concentrado en el rotavapor para dar un aceite marrón oscuro $(120 \mathrm{mg})$. La purificación se hizo por CC flash (gel de sílice, saturada con $\mathrm{Et}_{3} \mathrm{~N}$, Hex: $\mathrm{AcOEt}_{\mathrm{E}} \mathrm{Et}_{3} \mathrm{~N}$, 80:17:3) y se obtuvo la mezcla de diastereoisómeros 4:1, 31 y 32, siendo el compuesto 31 el isómero mayoritario, el cual fue purificado por CC flash (gel de sílice, saturada con $\mathrm{Et}_{3} \mathrm{~N}$, Hexano: AcOEt: $\left.\mathrm{Et}_{3} \mathrm{~N}, 90: 7: 3\right)$ y del cual se obtuvo (51.3 mg, 40.1\%) como un aceite incoloro.

Datos del isómero mayoritario 31: $\mathrm{RMN}^{-1} \mathrm{H}\left(300 \mathrm{MHz}, \mathrm{CDCl}_{3}\right) \delta 0.86(\mathrm{~s}, 3 \mathrm{H})$, $1.00(\mathrm{~s}, 3 \mathrm{H}), 1.46$ y $1.52(\mathrm{c} \mathrm{AB}, J=13.8 \mathrm{~Hz}, 2 \mathrm{H}), 2.10-2.45(\mathrm{~m}, 7 \mathrm{H}), 2.65(\mathrm{td}, J=8.6$, $5,7.0 \mathrm{~Hz}, 1 \mathrm{H}), 2.91(\mathrm{dd}, J=12.0,5.7 \mathrm{~Hz}, 1 \mathrm{H}), 3.42(\mathrm{~d}, J=13.2 \mathrm{~Hz}, 1 \mathrm{H}), 3.59(\mathrm{~d}, J=$ $13.2 \mathrm{~Hz}, 1 \mathrm{H}), 4.70(\mathrm{td}, J=7,6,6.2 \mathrm{~Hz}, 1 \mathrm{H}), 4.97(\mathrm{~s}, 1 \mathrm{H}), 5.30(\mathrm{~s}, 1 \mathrm{H}), 7.23-7.34(\mathrm{~m}, 5 \mathrm{H}$, Ar); RMN ${ }^{13} \mathrm{C}\left(75 \mathrm{MHz}, \mathrm{CDCl}_{3}\right), \delta 29.3\left(\mathrm{CH}_{3}\right), 29.9\left(\mathrm{CH}_{3}\right), 31.4\left(\mathrm{CH}_{2}\right), 36.2(\mathrm{C}), 42.9$ $(\mathrm{CH}), 46.7(\mathrm{C}), 49.8\left(\mathrm{CH}_{2}\right), 50.1\left(\mathrm{CH}_{2}\right), 53.9\left(\mathrm{CH}_{2}\right), 60.3\left(\mathrm{CH}_{2}\right), 62.4\left(\mathrm{CH}_{2}\right), 76.7(\mathrm{CH})$, $107.8\left(=\mathrm{CH}_{2}\right), 127.3(\mathrm{CH}), 128.3(\mathrm{CH}), 128.9(\mathrm{CH}), 137.7(\mathrm{C}), 157.9,176.3(\mathrm{C}=\mathrm{O}) . \mathrm{IR}$ (Neto) $1778(\mathrm{~s}, \mathrm{C}=\mathrm{O}), 1649(\mathrm{~m}, \mathrm{C}=\mathrm{C}), 1167(\mathrm{~m}, \mathrm{C}-\mathrm{O}) \mathrm{cm}^{-1} ; \mathrm{EM}(\mathrm{IE}) \mathrm{m} / \mathrm{z}(\%) 325$ (M, 8), 241 (25), 134 (31), 91 (base); EMAR calculado para: $\mathrm{C}_{21} \mathrm{H}_{27} \mathrm{NO}_{2} ; 325.2042$, observado: 325.2044 .

De la mezcla de $\mathbf{3 1} / \mathbf{3 2}$, se logró determinar los datos del isómero minoritario (32): $\mathrm{RMN}^{1} \mathrm{H}\left(300 \mathrm{MHz}, \mathrm{CDCl}_{3}\right), \delta 0.88(\mathrm{~s}, 3 \mathrm{H}), 1.06(\mathrm{~s}, 3 \mathrm{H}), 1.73-1.84(\mathrm{~m}, 2 \mathrm{H})$, 2.04-2.48 (m, 6H), 2.58-2.69 (m, 1H), $2.75(\mathrm{~d}, J=13.7 \mathrm{~Hz}, 1 \mathrm{H}), 3.08(\mathrm{dd}, J=11.7,6.4$ $\mathrm{Hz}, 1 \mathrm{H}), 3.95(\mathrm{~d}, J=13.6 \mathrm{~Hz}, 1 \mathrm{H}), 4.57-4.65(\mathrm{~m}, 1 \mathrm{H}), 4.88(\mathrm{br} \mathrm{s}, 1 \mathrm{H}), 5.06(\mathrm{br} \mathrm{s}, 1 \mathrm{H})$, 7.23-7.34 (m, 5H), RMN- ${ }^{13} \mathrm{C}(75 \mathrm{MHz}, \mathrm{CDCl} 3) \delta 24.7\left(\mathrm{CH}_{3}\right), 30.5\left(\mathrm{CH}_{2}\right), 32.2\left(\mathrm{CH}_{3}\right)$, $32.6(\mathrm{C}), 35.9(\mathrm{CH}), 39.7(\mathrm{CH}), 41.6\left(\mathrm{CH}_{2}\right), 44.0\left(\mathrm{CH}_{2}\right), 53.3\left(\mathrm{CH}_{2}\right), 57.7\left(\mathrm{CH}_{2}\right), 64.7$ $(\mathrm{CH}), 74.6(\mathrm{CH}), 114.4\left(\mathrm{CH}_{2}\right), 127.1(\mathrm{CH}), 128.4(\mathrm{CH}), 128.5(\mathrm{CH}), 139.1(\mathrm{C}), 146.3(\mathrm{C})$, $176.3(\mathrm{C})) ; \mathrm{CG} / \mathrm{EM} t \mathbf{R}(\mathbf{1 3 b})=20.6 \mathrm{~min}, t \mathbf{R}(\mathbf{1 4 b})=21.1 \mathrm{~min} ; \mathrm{EM}(\mathrm{EI}) \mathrm{m} / \mathrm{z}(\%) 325$ (M, 17), 242 (36), 91 (base). EMAR calculado para $\mathrm{C}_{21} \mathrm{H}_{27} \mathrm{NO}_{2} 325.2042$, observado: 325.2044 .

\section{Conclusiones}

Se pudo determinar la caracterización de 15 compuestos aislados en las especies E. alata y $R$. heptaphylla; de estos, cabe resaltar la identificación de alcaloides furoquinolínicos, piranoquinolínicos y cumarinas, característicos de estas especies y lo que está de acuerdo a la quimiotaxonomía de los géneros objeto de este estudio y de la familia Rutaceae.

De la especie $R$. heptaphylla se han encontrado pocos reportes, y los alcaloides que se han aislado en este trabajo se reportan por primera vez para esta especie, ya que anteriormente se han aislado alcaloides indólicos [4]. Para E. alata, a excepción de 
$\beta$-sitosterol y estigmasterol, los demás compuestos aislados se reportan por primera vez. Este es el primer reporte de lignanos en el género Esenbeckia, a pesar de la gran distribución de estos metabolitos en la familia Rutaceae.

Se desarrolló una posible ruta de síntesis para precursores de análogos de alcaloides quinolínicos, como es el caso de las tetrahidropiridinas formadas, las cuales presentan la tendencia en reacciones radicalarias a la formación de derivados Espiro prevaleciendo el camino 5-exo-trig, con un alto porcentaje de rendimiento, regio y estereoselectividad en los productos formados, siendo este el primer reporte de ciclaciones radicalarias partiendo de enaminas del tipo tetrahidropiridina, y no de derivados indólicos. Esta ruta de síntesis planteada permite la posibilidad de obtener una gran variedad de productos, usando diferentes sustratos de acuerdo al aldehido utilizado inicialmente.

\section{Agradecimientos}

Agradecemos a Colciencias, al grupo de Productos Naturales Vegetales de la Universidad Nacional de Colombia por la financiación del trabajo. Al profesor Jose Miguel Aurrecoechea de la Universidad del País Vasco por la asesoría y financiación en la parte de síntesis, y al Laboratorio de Resonancia Magnética Nuclear de la Universidad Nacional de Colombia por la toma de espectros, así como también a la Universidad Militar Nueva Granada por permitir validar las extracciones de los metabolitos de $R$. heptaphylla, a través de la financiación del proyecto CIAS1173.

\section{Referencias}

1. (a) Pollio, A.; De Natale, A.; Appetiti, E.; Aliotta, G.; Touwaide, A. Journal of Ethnopharmacology. 2008. 116. 469-482. (b) Cuca L.E. Tesis doctoral, Universidad Nacional de Colombia. Sede Bogotá 1999. 1-5.

2. (a) Rios, M.; Aguilar-Guadarrama, B. Biochemical Systematics and Ecology. 2002. 30. 977-979. (b) Rios, M.; Rosa-Alonso, E.; Aguilar-Guadarrama, B. Biochemical Systematics and Ecology. 2002. 32. 817-821. (c) Rios, M; Delgado, G. Journal of Natural Products. 1992. 55. 1307-1309.(d) Torres, A. Tesis de magíster, Departamento de Química, Universidad Nacional de Colombia. 2001. 10-15.

3. Bakhtiar, A.; Gleye, J.; Moulios, C.; Foraste, I. Phytochemistry. 1991. 30. 3840-3841.

4. Rosas, L.; André, T.; Das G.F.; Fernandes, J.; Vieira, P.; Oliva, G.; Thiemann, O. XXVI Reunião Anual Sobre Evolucão. Sistemática e Ecologia Micromoleculares Instituto de Química. Universidade Federal Fluminense, 1 a 3 de dezembro de 2004.

5. Alvarez, B.; Ramon-Laca, L. Journal of Ethnopharmacology. 2005. 97. 89-95.

6. Ito, C.; Itoigawa, M.; Onoda, S.; Hosokawa, A.; Ruangrungsi, N.; Okuda, T.; Tokuda, H.; Nishino, H.; Furukawa, H. Phytochemistry. 2005. 66. 567-572.

7. Chen, K.-S.; Chang, Y.-L.; Teng, C.-M.; Chen, C.; F.; and Wu, Y.-C. Planta Médica. 2000. 66. 80-81.

8. Hanawa, F.; Fokialakis, N.; Skaltsounis, A. Planta Medica. 2004. 70. 531-535.

9. Rahman, M., Gray, A... Phytochemistry. 2005. 13. 1601-1606.

10. Maximin K.; Ioset, J.; Ransijn, A.; Mauel, J.; Mavi, S.; Hostettmann, K. Phytochemistry. 2004. 65. 963-968.

11. Collet, I.; Benoit-Vical, F.; Mustofa, A.; Stanislas, E.; Mallie, M.; Fouraste, I. Planta Médica. 2001. 68. 68-69. 
12. Anaya, A.; Macias-Rubacava, M.; Cruz-Ortega, R.; García-Santana, C.; SánchezMonterrubio, P.; Hernández-Bautista, B.; Mata, R. Phytochemistry. 2005. 66. 487-494.

13. (a) Bertani, S. ; Bourdy, G. ; Landau, I. ; Robinson, J. ; Esterre, P. ; Deharo, E. Journal of Ethnopharmacology. 2005. 98. 45-54. (b) Dolabela, M.; Oliveira, S.; Nascimento, J.; Peres, J.M,; Wagner, H,; Povoa, M.; de Oliveira, A. Phytomedicine. 2008. 15. 367-372.

14. Michael, J.; Natural Products Reports. 2008. 25. 166-167.

15. (a) Aurrecoechea, J. M.; Gorgojo, J. M.; Saornil, C. Journal Organic Chemistry. 2005. 70. 9640-9643. (b) Aurrecoechea, J, M., Suero, R., De Torre, E.. Journal Organic Chemistry. 2006. 71. 8767-8777.

16. Reviews: (a) Parsons, A. F.C.R. Academic Sciences. II. 2001, 391-400. (b) Clarck, A.J. Journal Chemical Society. Reviews. 2002. 31. 1-12.

17. (a) Khan, F. A.; Dash, J.Journal Organic Chemistry. 2003. 68. 4556-4559.(b) Abe, H,; Aoyagi, S,; Kibayashi, S. Journal of the American Chemical Society. 2000. 122. 4583-4592.

18. (a) Parsons,; Penckett, C.S,; Cramp, M.C,; West, R.I,; Warrington, J,; Saraiva, M.C. Tetrahedron. 1996. 52.647-660.

19. Ahman, J,; Somfai, P,; Journal Chemical Society Perkin Trans I. 1994. 1072-1082.

20. (a) Yang, C. C,; Chang, H. T,; Fang, J.M. Journal Organic Chemistry. 1998. 58. 3100-3105. (b) Flanagan, S,; Harrowven, D.C,; Bradley, M. Tetrahedron Letters. 2003. 44.1795-1798.

21. Joucla, L,; Putey, A,; Joseph, B. Tetrahedron Letters. 2005. 46, 8177-8179.

22. (a) Coy, C.A and Cuca, L.E. Scientia et Technica. 2007. 33. 337-338. (b) Cuca, L.E and Coy, C.A. Biochemical Systematics and Ecology. 2007. 35. 386-388.

23. Hwu, J.; Tseng, W.; Gnabre, J.; Giza, P.; Huang, R. Journal of Medical Chemistry. 1998. 41. 2994-3000.

24. Pusset, J.; Lopez, J.; Neirabeyeh, M.; Veillon, J. Planta Medica. 1991. 57. 153-155.

25. Forgo, P.; Kuver, K. Steroids. 2004. 69.43-50.

26. Djilani, A., Legseir, B., Soulimani, R., Dicko, A., Younos, C. Journal of the Brazilian Chemical Society, 2006. 17. 518-520.

27. Merck. Reactivos de Coloración para Cromatografía en Capa Fina y en Papel. E. Merck, Darmstadt (R.F. de Alemania). 1965. 27-332.

28. Campbell, W,; Davidowitz, B,; Jackson, G. Phytochemistry. 1990. 4. 1304-1306.

29. Biavatti, M.; Vieira, P,; Da Silva, M,; Fernandes, J,; Victor, S,; Pagnocca, F,; Albuquerque, S,; Caracelli, I,; Scpector, J. Journal of the Brazilian Chemical Society. 2002.13. 66-70

30. Hon, Y., Chang, F., Lu, L., Lin, W. Tetrahedron. 1998. 5233-5246.

31. Ripa, L.; Hallberg, A. Journal of Organic Chemistry. 1998. 63.84- 91.

32. Brevion, F., Vitale, M., Fensterbank, L., Malacria, M. Tetrahedron Asymmetry. 2003. 14. 2889-2896.

33. Aurrecoechea, J. M.; Coy, C.A., Patiño, O. Journal of Organic Chemistry. 2008. 73, 5194-5197. 